\title{
The effects of perch cooling on performance, carcass, and meat quality characteristics and behaviour of broilers reared at high temperatures with different litter thicknesses
}

\author{
Evrim DERELİ FIDAN ${ }^{\mathrm{a}, \varpi}$, Mehmet Kenan TÜRKYILMAZ ${ }^{\mathrm{b}}$, Ahmet NAZLIGÜL ${ }^{\mathrm{c}}$, Mehmet KAYA ${ }^{\mathrm{d}}$ \\ Aydın Adnan Menderes University, Faculty of Veterinary Medicine, Department of Animal Science, Aydın, Turkey. \\ aORCID: 0000-0002-9805-6162; ' $\mathrm{ORCID:} \mathrm{0000-0002-7600-2390;} \mathrm{'} \mathrm{ORCID}$ : 0000-0003-1476-4039; \\ dORCID: 0000-0003-2377-4474. \\ Corresponding author: edereli@adu.edu.tr \\ Received date: 06.11.2019 - Accepted date: 25.03.2020
}

\begin{abstract}
The aim of the present study was to investigate the effects of cooled perch and litter thickness on performance, meat quality, carcass, and behaviour traits on broilers reared at high environmental temperatures. A total of 4591 day-old male broiler chicks were randomly assigned to nine treatment groups based on the perch treatment (no perch, non-cooled perches, cooled perches) and litter thicknesses $(1,7$ and $14 \mathrm{~cm})$ with three replicates. Body weight was higher in broilers reared at cooled perch group at 6 wks $(\mathrm{P}<0.05)$. Body weight of chickens reared under the $1 \mathrm{~cm}$ thickness was found higher than the 7 and $14 \mathrm{~cm}$ of litter thickness chickens at 6 wks. Live and carcass weights, total breast and drum weights were higher in broilers reared at $1 \mathrm{~cm}$ litter thickness. The perchcontact incidence of chickens reared under the cooled perch was found higher than the non-cooled chickens for all hours at 6 wks. In conclusion, it is indicated that perch cooling and $1 \mathrm{~cm}$ of litter thickness have a beneficial effect on broiler performance exposed to high temperatures.
\end{abstract}

Keywords: Broiler, cool perch, meat quality, perching, performance

\section{Tünek soğutmanın farklı altık kalınlıklarında yüksek sıcaklıklarda yetiştirilen etlik piliçlerin performans, karkas ve et kalite özellikleri ile davranışları üzerine etkileri}

Özet: Bu çalışmanın amacı yüksek çevresel sıcaklıklarda yetiştirilen etlik piliçlerde soğutulmuş tünek kullanımı ve altlık kalınlığının performans, et kalitesi, karkas ve davranış özellikleri üzerine etkilerini araştırmaktır. Toplam 459 bir günlük yaşta erkek etlik piliçler, tünek denemesi (tünek yok, soğutma olmayan ve soğuk tünek) ve altlık kalınlıklarına (1, 7 ve $14 \mathrm{~cm})$ rastgele 3 tekrarlı olacak şekilde 9 gruba yerleştirilmiştir. Soğutulmuş tünek grubunda yetiştirilen etlik piliçlerde 6 . haftada canlı ağırlık en yüksek değerde bulunmuştur. $1 \mathrm{~cm}$ altlık kalınlığı grubunda yetiştirilen etlik piliçlerde altıncı haftada canlı ağırlığ 7 ve $14 \mathrm{~cm}$ kalınlık grubunda yetiştirilen tavuklara göre daha yüksek bulunmuştur. Kesim öncesi canlı ağırlığı ve karkas ağırlığı, bütün göğüs ve kalçalı but ağırlıkları $1 \mathrm{~cm}$ altlık kalınlığı grubunda daha yüksek bulunmuştur. Altıncı haftada ve incelenen tüm saatler için etlik piliçlerin tünek ile teması bakımından soğutulmuş tünekler ile temas sıklığı soğutulmamış tünek temasına göre daha yüksektir. Sonuç olarak, yüksek sıcaklıklara maruz kalan etlik piliçlerde soğuk tünek ve $1 \mathrm{~cm}$ altlık kalınlığının etlik piliç performansı üzerine olumlu etkisinin olduğu belirlenmiştir.

Anahtar sözcükler: Et kalitesi, etlik piliç, performans, soğuk tünek, tüneme

\section{Introduction}

Broiler chicken production has increased gradually and become one of the most intense animal production systems. Perching is an extremely moved behaviour in domestic chickens (18). However, the rate of perch use in broiler chickens is lower than in laying hens (19). The reason for this may be due to fast growth rate and therefore increased weight gain and higher body weight (BW) in early ages, and less physical activity of broilers in the following weeks (17). Broilers prefer to perch on cooled perch when exposed to hot weather, because they heat elimination of body by conduction $(7,21,26)$. And, the cooled perch use contributes positively to the short life of modern lines of broiler chicks. Therefore, recent studies relating the perch have focused on providing a cooler microclimate in hot weather (such as cooled perch). Reilly et al. (21) in broilers and Muiruri and Harrison (16) in broilers breeder hens reported that exposed to high temperatures to provide perching on cooled perches, resulting in bird performance. It has been suggested that 
litter thickness of broilers is $1 \mathrm{~cm}$ in high ambient temperatures (6). The effect of litter thickness (from 4 to $16 \mathrm{~cm}$ ) on BW, BW gain, FI, and liver weight in broiler chickens is reported as statistically significant by a study (23).

The aim of this study was to investigate the effects of cooled perches and three different litter thickness on performance, processing characteristics, and meat quality traits of broilers reared in summer. Additionally, daytime behavioural patterns were also indicated.

\section{Material and Methods}

Birds and husbandry: This study was carried out at the Poultry Breeding Unit of Aydin Adnan Menderes University. The study was performed with the permission of the Aydin Adnan Menderes University Animal Experimentation Local Ethics Committee (13.06.201764583101/049). A total of 459 1-d-old male broiler chicks were randomly assigned to perch and litter thickness treatments with three replications as to $3 \times 3$ factorial arrangement of 3 perch treatments (no perch, non-cooled and cooled perches) and 3 litter thickness treatments (1, 7, and $14 \mathrm{~cm}$ litter thicknesses of pine wood shavings). Chicks of each treatment were placed in separate floor pens (in a total of 27 pens $(3 \times 3=9$ main groups, $9 \times 3$ replicates $=27$ pens) measuring $110 \times 150 \mathrm{~cm}$, width $\mathrm{x}$ length, respectively. All chicks were fed with balanced diets (1-14 d; $3050 \mathrm{kcal} \mathrm{ME} / \mathrm{kg}, 23.5 \%$ crude protein, 15 $28 \mathrm{~d} ; 3150 \mathrm{kcal} \mathrm{ME} / \mathrm{kg}, 22 \%$ crude protein, and 29-42 d; $3200 \mathrm{kcal} \mathrm{ME} / \mathrm{kg}, 21 \%$ crude protein) and watered ad libitum. The photoperiod lengths were applied as continuous lighting ( $24 \mathrm{~h}$ light) for 1 to $7 \mathrm{~d}$ and 40 to 42 days and 18L: $6 \mathrm{D}$ for 8 to 39 days (8). The brooding temperature was set to $34^{\circ} \mathrm{C}$ for the first $3 \mathrm{~d}$ and was reduced gradually to $31^{\circ} \mathrm{C}$ at the end of the week for all pens. Mechanical ventilation was controlled separately by a chamber with exhaust fans running 15 min on and 120 min off to 2 wks and $15 \mathrm{~min}$ on and $60 \mathrm{~min}$ off thereafter. From 2 to $6 \mathrm{wks}$ of age, exhaust fans overrode the timer when chamber ambient temperature reached. The air temperature and relative humidity were recorded continuously in three median points of each pen, at the height of the bird's eye. Measurements were performed for four times in a day by using dataloggers at 08:00, 12:00, 16:00 and 20:00 hours throughout the experiment.

Experimental design: The perches used in noncooled and cooled treatments were identical and consisted of galvanized steel pipes $(1.1 \mathrm{~m}$ length, $3.0 \mathrm{~cm}$ in diameter). In terms of perch application, 18 steel perches (9 cooled and 9 non-cooled) were horizontally attached 10 $\mathrm{cm}$ above the pen litter as to experimental design. The position of both cooled and non-cooled perches was set to make chicks to pass over the perch for feeding and drinking. For cooling 9 out of the 18 perches were connected to the water cooler (Cihso 2000, Cihan Cooling Ltd. Co., Aydın, Turkey), which has capable of cool, and circulate $5 \mathrm{~L} / \mathrm{min}$ water at $10^{\circ} \mathrm{C}$ through the pipes. Water temperature in cooled perches was controlled twice daily. Chickens were exposed to perches from $1 \mathrm{~d}$, and the cooling process was initiated on at $14 \mathrm{~d}$. Cooling was provided for the rest of the experimental period.

Traits measured: The BW gain and feed intake (FI) were weekly measured on a per replicate basis. Feed conversion ratio (FCR) of related periods was calculated. Broiler mortality was recorded for each pen on daily basis from which the total mortality ratio was calculated (0-42 d). On 42 d, seven chickens from each experiment pen were randomly selected for processing performance and meat quality traits. Feed was withdrawn $12 \mathrm{~h}$ before slaughter. Broilers shackled, and killed by cut severing jugular vein and the right carotid artery, and exsanguinated. Total carcasses (without giblets, neck) were then drained, weighed, and deboned to obtain with skin, boneless total breast meat (fillets and tenders) and legs (thighs and drums). Carcass parts were weighted and expressed as percentages of carcass weight. In the study, breast and thigh meats were used to assign meat quality traits. The $\mathrm{pH}$ of breast and thigh meats were measured at 15 min postmortem $\left(\mathrm{pH}_{15}\right)$ and $24 \mathrm{~h}$ after slaughter $\left(\mathrm{pH}_{\mathrm{u}}\right)$ using a digital pH meter (Hanna Inst. No: HI 9124). Meat color was measured on the surface on left breast thigh meats at $24 \mathrm{~h}$ postmortem, and this process was performed by a Minolta CR-400 color meter (Konica Minolta Sensing, Inc., Osaka, Japan) in the CIELAB color space using a D65 illuminant. Values of $\mathrm{L}^{*}, \mathrm{a}^{*}$, and $\mathrm{b}^{*}$ indicate lightness, redness, and yellowness, respectively. A cooking loss (CL) was determined in breast and thigh meat samples accordingly to the traditional method of Honikel (11). CL was expressed as the percentage of loss related to the initial weight. Water holding capacity (WHC) was evaluated at $24 \mathrm{~h}$ postmortem, using the methodology described by Barton-Gade et al. (3). The suggested methods of the standard analytical methods Association of Official Analytical Chemists (1) were analyzed for the determination of breast muscle chemical composition (moisture, crude fat, protein, and ash). Daily perching behaviors of birds were monitored and recorded from the days of 35 to 42 with cameras connected to the recording system which were placed on top of each pen. These camera recordings were investigated for 4 hours intervals (6 times a day) for three minutes. In other words, $18 \mathrm{~min}$ of recording period for each day was evaluated. In this assessment, if the bird was standing, sitting, or walking with both feet on the perch and staying at least $2 \mathrm{~s}$, the bird was defined as a perching (27). However, if the chicken wasn't perching, but was in physical contact with the perch (perch-contacting) (e.g., staying bird's body parts such as head, neck, breast, or wing on the perch), these 
animals were evaluated as animals being in contact with the perch. No perching and perch-contacting behaviours were accepted as other behaviours (e.g. eating, drinking, walking, and resting on the floor).

Statistical analyses: Data were analyzed using the Statistical Package for the Social Sciences for Windows (SPSS) 22.0 (SPSS Inc, Chicago, IL. USA). Analysis of variance was performed with GLM procedure to reveal the effects of cooled perch and litter thickness on performance and carcass characteristics, meat quality traits. Multiple comparisons were achieved by Duncan's multiple range test. Chi-square test was performed for perching behavior and mortality.

\section{Results}

In study, maximum diurnal ambient temperatures for dry bulb in the house from three and six weeks of age altered from 31.4 to $34.2^{\circ} \mathrm{C}$, which are characteristic for Aydin region in Turkey. The BW was found the highest in cooled perch $(\mathrm{P}<0.001)$ and $1 \mathrm{~cm}$ litter thickness groups $(\mathrm{P}<0.001)$ at $42 \mathrm{~d}$ (Table 1). Litter thickness affected cumulative FI $(\mathrm{P}<0.05)$ of broilers from 0 to 42 days of age. There was no significant between litter thickness groups for FCR. The differences between 1, 7, and $14 \mathrm{~cm}$ litter thickness groups for mortality rate were not significant (Table 2). Litter thickness significantly affected the weights of the carcass, total breast and legs, and drums

Table 1. Influences of cooled perch and litter thickness on body weight of broilers ${ }^{1}$.

\begin{tabular}{|c|c|c|c|c|c|c|c|c|c|c|c|c|c|c|}
\hline \multirow[b]{2}{*}{$\begin{array}{l}\text { Treatment main } \\
\text { effects }\end{array}$} & \multicolumn{14}{|c|}{ Body weight (g) } \\
\hline & $\mathbf{n}$ & d 1 & $\mathbf{n}$ & d 7 & $\mathbf{n}$ & d 14 & $\mathbf{n}$ & d 21 & $\mathbf{n}$ & d 28 & $\mathbf{n}$ & d 35 & $\mathbf{n}$ & d 42 \\
\hline $\begin{array}{l}\text { Expected mean } \\
(\mu)\end{array}$ & 459 & 45.27 & 459 & 145.97 & 459 & 384.25 & 459 & 813.83 & 459 & 1367.17 & 455 & 2040.04 & 452 & 2566.54 \\
\hline \multicolumn{15}{|l|}{ Perch treatment } \\
\hline No perch & 153 & 45.13 & 153 & 143.77 & 153 & $387.95^{\mathrm{a}}$ & 153 & 812.98 & 153 & $1350.86^{\mathrm{b}}$ & 151 & $1990.41^{\mathrm{c}}$ & 151 & $2530.15^{\mathrm{b}}$ \\
\hline $\begin{array}{l}\text { Non-cooled } \\
\text { perch }\end{array}$ & 153 & 45.76 & 153 & 145.90 & 153 & $393.02^{\mathrm{a}}$ & 153 & 815.93 & 153 & $1363.34^{\mathrm{ab}}$ & 152 & $2034.82^{b}$ & 151 & $2519.89^{b}$ \\
\hline Cooled perch & 153 & 44.91 & 153 & 148.25 & 153 & $371.78^{\mathrm{b}}$ & 153 & 812.57 & 153 & $1387.30^{\mathrm{a}}$ & 152 & $2094.90^{\mathrm{a}}$ & 150 & $2649.57^{\mathrm{a}}$ \\
\hline \multicolumn{15}{|l|}{ Litter thickness } \\
\hline $1 \mathrm{~cm}$ & 153 & 44.86 & 153 & $148.48^{\mathrm{a}}$ & 153 & 389.58 & 153 & 824.90 & 153 & $1388.37^{\mathrm{a}}$ & 153 & $2104.58^{\mathrm{a}}$ & 153 & $2662.51^{\mathrm{a}}$ \\
\hline $7 \mathrm{~cm}$ & 153 & 45.59 & 153 & $143.00^{\mathrm{b}}$ & 153 & 377.25 & 153 & 810.45 & 153 & $1351.09^{\mathrm{b}}$ & 151 & $2008.74^{\mathrm{b}}$ & 150 & $2526.41^{\mathrm{b}}$ \\
\hline $14 \mathrm{~cm}$ & 153 & 45.35 & 153 & $146.44^{\mathrm{ab}}$ & 153 & 385.91 & 153 & 806.14 & 153 & $1362.03^{\mathrm{ab}}$ & 151 & $2006.82^{b}$ & 149 & $2510.69^{b}$ \\
\hline $\mathrm{SEM}^{2}$ & & 0.18 & & 0.83 & & 2.16 & & 3.84 & & 6.01 & & 8.62 & & 11.52 \\
\hline Treatment effects & & & & & & & & P value & & & & & & \\
\hline Perch & & 0.124 & & 0.089 & & 0.000 & & 0.927 & & 0.043 & & 0.000 & & 0.000 \\
\hline Litter thickness & & 0.219 & & 0.025 & & 0.057 & & 0.114 & & 0.035 & & 0.000 & & 0.000 \\
\hline
\end{tabular}

${ }^{1}$ : Data presented as the least square means, ${ }^{\text {a, b, c }}$ : Means with different superscript letters in the same column $\operatorname{differ}(\mathrm{P}<0.05),{ }^{2}:$ Standard error mean.

Table 2. Effect of perch treatment and litter thickness on cumulative feed consumption, feed conversion, and mortality of broilers.

\begin{tabular}{|c|c|c|c|c|c|c|c|c|c|c|c|c|c|c|}
\hline \multirow[b]{2}{*}{$\begin{array}{l}\text { Treatment } \\
\text { main effects }\end{array}$} & \multirow[b]{2}{*}{$\mathbf{n}$} & \multicolumn{6}{|c|}{ Cumulative feed intake (g/bird) } & \multicolumn{6}{|c|}{$\begin{array}{c}\text { Cumulative feed conversion ratio } \\
\text { ( } \mathrm{g} \text { of feed } / \mathrm{g} \text { of gain) }\end{array}$} & \multirow{2}{*}{$\begin{array}{c}\text { Total } \\
\text { mortality } \\
(\%)\end{array}$} \\
\hline & & d 0-7 & d 0-14 & d 0-21 & d 0-28 & d 0-35 & d 0-42 & d 0-7 & d 0-14 & d 0-21 & d 0-28 & d $\mathbf{0 - 3 5}$ & d 0-42 & \\
\hline $\begin{array}{l}\text { Expected } \\
\text { mean }(\mu)\end{array}$ & 27 & 191.14 & 555.89 & 1120.53 & 1961.83 & 3095.06 & 4178.19 & 1.91 & 1.65 & 1.46 & 1.48 & 1.57 & 1.69 & 1.5 \\
\hline \multicolumn{15}{|c|}{ Perch treatment } \\
\hline No perch & 9 & $199.86^{\mathrm{a}}$ & 570.18 & 1134.63 & 1971.41 & 3079.24 & 4148.56 & $2.04^{\mathrm{a}}$ & 1.67 & 1.48 & $1.51^{\mathrm{a}}$ & $1.61^{\mathrm{a}}$ & 1.69 & 1.3 \\
\hline $\begin{array}{l}\text { Non-cooled } \\
\text { perch }\end{array}$ & 9 & $193.04^{\mathrm{a}}$ & 558.90 & 1119.70 & 1965.46 & 3096.86 & 4155.52 & $1.94^{\mathrm{ab}}$ & 1.61 & 1.45 & $1.49^{\mathrm{a}}$ & $1.57^{\mathrm{ab}}$ & 1.71 & 1.3 \\
\hline Cooled perch & 9 & $180.52^{b}$ & 538.59 & 1107.25 & 1948.61 & 3109.10 & 4230.50 & $1.75^{\mathrm{b}}$ & 1.66 & 1.44 & $1.45^{\mathrm{b}}$ & $1.53^{\mathrm{b}}$ & 1.66 & 2.0 \\
\hline \multicolumn{15}{|c|}{ Litter thickness } \\
\hline $1 \mathrm{~cm}$ & 9 & 187.66 & 553.71 & 1125.21 & 1979.35 & $3147.13^{\mathrm{a}}$ & $4286.31^{\mathrm{a}}$ & 1.82 & 1.61 & 1.44 & 1.47 & $1.53^{\mathrm{b}}$ & 1.64 & 0.0 \\
\hline $7 \mathrm{~cm}$ & 9 & 194.67 & 572.61 & 1135.13 & 1971.23 & $3092.76^{\mathrm{ab}}$ & $4150.25^{\mathrm{b}}$ & 2.01 & 1.73 & 1.48 & 1.51 & $1.60^{\mathrm{a}}$ & 1.71 & 2.0 \\
\hline $14 \mathrm{~cm}$ & 9 & 191.08 & 541.34 & 1101.24 & 1934.90 & $3045.30^{\mathrm{b}}$ & $4098.03^{\mathrm{b}}$ & 1.90 & 1.60 & 1.45 & 1.47 & $1.58^{\mathrm{ab}}$ & 1.71 & 2.6 \\
\hline SEM $^{1}$ & & 2.38 & 9.26 & 8.81 & 9.98 & 14.53 & 23.45 & 0.04 & 0.04 & 0.01 & 0.01 & 0.01 & 0.01 & \\
\hline \multicolumn{2}{|c|}{ Treatment effects } & \multicolumn{6}{|c|}{ P value } & \multicolumn{7}{|c|}{$P$ value } \\
\hline Perch & & 0.012 & 0.389 & 0.462 & 0.634 & 0.705 & 0.310 & 0.020 & 0.799 & 0.504 & 0.012 & 0.031 & 0.355 & 0.865 \\
\hline $\begin{array}{l}\text { Litter } \\
\text { thickness }\end{array}$ & & 0.498 & 0.400 & 0.296 & 0.182 & 0.034 & 0.012 & 0.137 & 0.265 & 0.364 & 0.089 & 0.047 & 0.060 & 0.152 \\
\hline
\end{tabular}

a, b: Means with different superscript letters in the same column differ $(\mathrm{P}<0.05),{ }^{1}$ : Standard error mean. 
weights $(\mathrm{P}<0.05)$. The cooled perch and litter thickness have statistically not significant effect on protein, fat, moisture, ash contents of breast meat (Table 3). Compared with the cooled perch group, no perch decreases FPD in
$\mathrm{L}^{*}$ value of thigh meat (Table 4). A higher proportion of chickens in perch-contacting in cooled perch group for all hours at 6 wks (Table 5).

Table 3. The least square means for live weight, carcass parameters, carcass part weights, protein, fat, moisture, and ash values of breast meat in treatment groups.

\begin{tabular}{|c|c|c|c|c|c|c|c|c|c|c|}
\hline $\begin{array}{l}\text { Treatment main } \\
\text { effects }\end{array}$ & $\begin{array}{c}\text { Live } \\
\text { weight (g) }\end{array}$ & $\begin{array}{c}\text { Carcass } \\
\text { weight } \\
\text { (g) }\end{array}$ & $\begin{array}{c}\text { Total breast } \\
\text { weight (g) }\end{array}$ & $\begin{array}{c}\text { Total leg } \\
\text { weight }(\mathrm{g})\end{array}$ & $\begin{array}{c}\text { Thighs } \\
\text { weight (g) }\end{array}$ & $\begin{array}{l}\text { Drums } \\
\text { weight } \\
\text { (g) }\end{array}$ & $\begin{array}{c}\text { Crude } \\
\text { protein } \\
(\%)\end{array}$ & $\begin{array}{l}\text { Crude } \\
\text { fat }(\%)\end{array}$ & $\begin{array}{c}\text { Crude } \\
\text { moisture } \\
(\%)\end{array}$ & $\begin{array}{l}\text { Crude } \\
\text { ash }(\%)\end{array}$ \\
\hline Expected mean $(\mu)$ & 2555.21 & 1958.16 & 601.71 & 572.14 & 296.57 & 275.57 & 19.47 & 2.99 & 74.87 & 1.05 \\
\hline \multicolumn{11}{|l|}{ Perch treatment } \\
\hline No perch & 2560.06 & 1959.56 & 594.11 & 580.40 & 302.46 & 277.94 & 19.51 & 3.23 & 74.74 & 0.97 \\
\hline Non-cooled perch & 2522.79 & 1949.92 & 592.59 & 575.27 & 299.60 & 275.67 & 19.53 & 3.28 & 74.79 & 1.13 \\
\hline Cooled perch & 2582.76 & 1965.00 & 618.43 & 560.75 & 287.65 & 273.10 & 19.37 & 2.47 & 75.08 & 1.05 \\
\hline \multicolumn{11}{|l|}{ Litter thickness } \\
\hline $1 \mathrm{~cm}$ & $2641.59^{\mathrm{a}}$ & $2006.71^{\mathrm{a}}$ & $623.71^{\mathrm{a}}$ & 581.92 & 299.73 & $282.19^{\mathrm{a}}$ & 19.37 & 3.07 & 74.44 & 0.97 \\
\hline $7 \mathrm{~cm}$ & $2499.64^{b}$ & $1920.84^{\mathrm{b}}$ & $583.02^{\mathrm{b}}$ & 568.78 & 295.52 & $273.25^{\mathrm{a}, \mathrm{b}}$ & 19.54 & 2.59 & 75.10 & 1.04 \\
\hline $14 \mathrm{~cm}$ & $2524.40^{\mathrm{b}}$ & $1946.92^{\mathrm{a}, \mathrm{b}}$ & $598.40^{\mathrm{a}, \mathrm{b}}$ & 565.71 & 294.46 & $271.25^{\mathrm{b}}$ & 19.50 & 3.32 & 75.06 & 1.14 \\
\hline SEM $^{1}$ & 16.16 & 13.34 & 6.02 & 4.04 & 2.69 & 1.87 & 0.20 & 0.23 & 0.20 & 0.05 \\
\hline Treatment effects & & & & & $P$ value & & & & & \\
\hline Perch & 0.312 & 0.897 & 0.148 & 0.123 & 0.060 & 0.573 & 0.936 & 0.304 & 0.754 & 0.438 \\
\hline Litter thickness & 0.001 & 0.028 & 0.023 & 0.223 & 0.699 & 0.042 & 0.940 & 0.447 & 0.332 & 0.400 \\
\hline
\end{tabular}

a, b: Means with different superscript letters in the same column differ $(\mathrm{P}<0.05),{ }^{1}$ : Standard error mean.

Table 4. Effects of cooled perch and litter thickness on breast and thigh meat quality traits of broiler chickens ${ }^{1}$.

\begin{tabular}{|c|c|c|c|c|c|c|c|c|c|c|c|c|c|c|}
\hline \multirow[b]{2}{*}{$\begin{array}{l}\text { Treatment main } \\
\text { effects }\end{array}$} & \multicolumn{7}{|c|}{ Breast meat } & \multicolumn{7}{|c|}{ Thigh meat } \\
\hline & $\mathrm{pH}_{15}$ & $\mathbf{p H}_{\mathrm{u}}$ & $\mathbf{L}^{*}$ & $\mathbf{a}^{*}$ & $\mathbf{b}^{*}$ & $\begin{array}{l}\text { CL } \\
(\%)\end{array}$ & $\begin{array}{c}\text { WHC } \\
(\%)\end{array}$ & $\mathrm{pH}_{15}$ & $\mathbf{p H}_{\mathrm{u}}$ & $\mathbf{L}^{*}$ & $\mathbf{a}^{*}$ & $\mathbf{b}^{*}$ & $\begin{array}{l}\text { CL } \\
(\%)\end{array}$ & $\begin{array}{c}\text { WHC } \\
(\%)\end{array}$ \\
\hline Expected mean $(\mu)$ & 6.73 & 6.13 & 54.11 & 1.00 & 4.02 & 29.29 & 7.47 & 6.66 & 6.46 & 59.30 & 7.63 & 8.14 & 38.53 & 6.83 \\
\hline \multicolumn{15}{|l|}{ Perch treatment } \\
\hline No perch & 6.78 & 6.08 & 54.25 & 1.27 & 3.64 & 33.55 & 8.70 & $6.74^{\mathrm{a}}$ & 6.48 & $62.54^{\mathrm{a}}$ & 7.06 & 7.84 & 36.69 & 6.52 \\
\hline Non-cooled perch & 6.70 & 6.07 & 53.62 & 0.66 & 4.26 & 27.50 & 6.23 & $6.56^{\mathrm{b}}$ & 6.37 & $58.71^{\mathrm{a}, \mathrm{b}}$ & 8.43 & 8.66 & 42.90 & 8.96 \\
\hline Cooled perch & 6.73 & 6.27 & 54.71 & 1.22 & 4.04 & 27.69 & 8.10 & $6.71^{\mathrm{a}}$ & 6.56 & $56.96^{\mathrm{b}}$ & 6.98 & 7.68 & 33.82 & 8.41 \\
\hline \multicolumn{15}{|l|}{ Litter thickness } \\
\hline $1 \mathrm{~cm}$ & 6.75 & 6.10 & 54.82 & 1.29 & 4.12 & 30.28 & 7.83 & 6.62 & 6.52 & $59.33^{\mathrm{a}, \mathrm{b}}$ & 8.98 & 9.39 & 40.61 & 6.55 \\
\hline $7 \mathrm{~cm}$ & 6.76 & 6.19 & 52.79 & 0.89 & 3.94 & 29.09 & 6.96 & 6.70 & 6.47 & $56.27^{\mathrm{b}}$ & 6.72 & 6.60 & 35.00 & 6.44 \\
\hline $14 \mathrm{~cm}$ & 6.61 & 6.01 & 55.95 & 0.46 & 3.99 & 26.90 & 7.93 & 6.66 & 6.22 & $68.31^{\mathrm{a}}$ & 6.26 & 9.02 & 42.90 & 8.79 \\
\hline $\mathrm{SEM}^{2}$ & 0.03 & 0.04 & 0.57 & 0.25 & 0.38 & 2.22 & 0.62 & 0.03 & 0.04 & 0.93 & 0.50 & 0.66 & 2.11 & 0.57 \\
\hline Treatment effects & \multicolumn{7}{|c|}{$P$ value } & \multicolumn{7}{|c|}{$P$ value } \\
\hline Perch & 0.687 & 0.128 & 0.391 & 0.756 & 0.732 & 0.359 & 0.206 & 0.033 & 0.444 & 0.010 & 0.213 & 0.909 & 0.328 & 0.068 \\
\hline Litter thickness & 0.637 & 0.608 & 0.122 & 0.706 & 0.944 & 0.962 & 0.517 & 0.208 & 0.385 & 0.008 & 0.066 & 0.163 & 0.454 & 0.254 \\
\hline
\end{tabular}

${ }^{1}$ : Data presented as the least square means, ${ }^{\text {a, }}$ : Means with different superscript letters in the same column differ $(\mathrm{P}<0.05), \mathrm{L}^{*}$ : Lightness, $\mathrm{a}^{*}$ : Redness, $\mathrm{b}^{*}$ : Yellowness, CL: Cooking loss, WHC: Water holding capacity, ${ }^{2}$ : Standard error mean. 
Table 5. Effect of cooled perch and litter thickness on the incidence (\%) of perching, perch-contacting and other behaviors in broilers at 6 wks of age.

\begin{tabular}{|c|c|c|c|c|c|c|c|c|c|c|c|c|c|c|c|c|c|c|}
\hline \multicolumn{19}{|c|}{ Incidence of perching, perch-contacting, and other behavioral (\%) } \\
\hline \multirow[b]{3}{*}{ Factors } & \multirow{2}{*}{\multicolumn{6}{|c|}{$\begin{array}{l}\text { Perching } \\
\text { Hours }\end{array}$}} & \multirow{2}{*}{\multicolumn{6}{|c|}{$\begin{array}{c}\text { Perch-contacting } \\
\text { Hours }\end{array}$}} & \multirow{2}{*}{\multicolumn{6}{|c|}{$\begin{array}{c}\text { Other behaviors } \\
\text { Hours }\end{array}$}} \\
\hline & & & & & & & & & & & & & & & & & & \\
\hline & $\mathbf{0}$ & 4 & 8 & 12 & 16 & 20 & $\mathbf{0}$ & 4 & 8 & 12 & 16 & 20 & $\mathbf{0}$ & 4 & 8 & 12 & 16 & 20 \\
\hline \multicolumn{19}{|c|}{ Perch treatment } \\
\hline $\begin{array}{l}\text { Non-cooled } \\
\text { perch }\end{array}$ & $7.5^{\mathrm{a}}$ & $6.6^{\mathrm{a}}$ & $6.2^{\mathrm{a}}$ & $6.9^{\mathrm{a}}$ & $9.3^{\mathrm{a}}$ & $8.3^{\mathrm{a}}$ & $15.6^{\mathrm{a}}$ & $11.2^{\mathrm{a}}$ & $11.3^{\mathrm{a}}$ & $4.8^{\mathrm{a}}$ & $16.3^{\mathrm{a}}$ & $13.1^{\mathrm{a}}$ & $76.9^{\mathrm{a}}$ & $82.2^{\mathrm{a}}$ & $82.5^{\mathrm{a}}$ & $78.3^{\mathrm{a}}$ & $74.4^{\mathrm{a}}$ & $78.6^{\mathrm{a}}$ \\
\hline $\begin{array}{l}\text { Cooled } \\
\text { perch }\end{array}$ & $7.4^{\mathrm{b}}$ & $4.9^{\mathrm{b}}$ & $5.2^{\mathrm{b}}$ & $4.3^{\mathrm{b}}$ & $4.8^{\mathrm{b}}$ & $5.3^{\mathrm{b}}$ & $39.3^{\mathrm{b}}$ & $41.3^{\mathrm{b}}$ & $36.1^{\mathrm{b}}$ & $9.2^{\mathrm{b}}$ & $40.8^{\mathrm{b}}$ & $38.8^{\mathrm{b}}$ & $53.3^{\mathrm{b}}$ & $53.8^{\mathrm{b}}$ & $58.8^{\mathrm{b}}$ & $56.5^{\mathrm{b}}$ & $54.5^{\mathrm{b}}$ & $55.9^{\mathrm{b}}$ \\
\hline \multicolumn{19}{|c|}{ Litter thickness } \\
\hline $1 \mathrm{~cm}$ & $10.3^{\mathrm{a}}$ & $7.0^{\mathrm{a}}$ & $7.6^{\mathrm{a}}$ & $7.4^{\mathrm{a}}$ & $7.3^{\mathrm{a}}$ & $7.8^{\mathrm{a}}$ & $25.1^{\mathrm{b}}$ & $25.0^{\mathrm{b}}$ & $23.4^{\mathrm{b}}$ & $25.0^{\mathrm{b}}$ & $27.8^{\mathrm{b}}$ & $24.8^{\mathrm{b}}$ & $64.6^{\mathrm{a}}$ & $68.0^{\mathrm{a}}$ & $68.9^{\mathrm{a}}$ & $67.6^{\mathrm{a}}$ & $64.9^{\mathrm{a}}$ & $67.5^{\mathrm{a}}$ \\
\hline $7 \mathrm{~cm}$ & $6.4^{\mathrm{b}}$ & $5.4^{\mathrm{a}}$ & $4.1^{\mathrm{b}}$ & $3.5^{\mathrm{b}}$ & $6.9^{\mathrm{a}}$ & $6.3^{\mathrm{a}}$ & $36.2^{\mathrm{a}}$ & $37.2^{\mathrm{a}}$ & $30.8^{\mathrm{a}}$ & $37.7^{\mathrm{a}}$ & $35.7^{\mathrm{a}}$ & $34.9^{\mathrm{a}}$ & $57.4^{\mathrm{b}}$ & $57.4^{\mathrm{b}}$ & $65.1^{\mathrm{a}, \mathrm{b}}$ & $58.7^{\mathrm{b}}$ & $57.4^{\mathrm{b}}$ & $58.9^{\mathrm{b}}$ \\
\hline $14 \mathrm{~cm}$ & $1.1^{\mathrm{c}}$ & $3.3^{\mathrm{b}}$ & $2.1^{\mathrm{b}}$ & $1.6^{\mathrm{b}}$ & $1.6^{\mathrm{b}}$ & $1.5^{\mathrm{b}}$ & $41.7^{\mathrm{a}}$ & $39.8^{\mathrm{a}}$ & $36.4^{\mathrm{a}}$ & $36.9^{\mathrm{a}}$ & $41.7^{\mathrm{a}}$ & $38.2^{\mathrm{a}}$ & $57.2^{\mathrm{b}}$ & $9.1^{\mathrm{b}}$ & $61.5^{\mathrm{b}}$ & $61.5^{\mathrm{a}, \mathrm{b}}$ & $56.7^{\mathrm{b}}$ & $60.3^{\mathrm{b}}$ \\
\hline Hours & & & \multicolumn{3}{|c|}{0} & \multicolumn{2}{|l|}{4} & \multicolumn{2}{|c|}{8} & & \multicolumn{2}{|c|}{12} & \multicolumn{2}{|c|}{16} & \multicolumn{4}{|c|}{20} \\
\hline $\mathrm{X}^{2}$ & & & \multicolumn{3}{|c|}{69.891} & \multicolumn{2}{|l|}{57.091} & \multicolumn{2}{|c|}{41.258} & & \multicolumn{2}{|c|}{57.302} & \multicolumn{2}{|c|}{40.493} & \multicolumn{4}{|c|}{45.524} \\
\hline $\mathrm{P}$ & & & \multicolumn{2}{|c|}{$* * *$} & & \multicolumn{2}{|l|}{$* * *$} & \multicolumn{2}{|c|}{$* * *$} & & \multicolumn{2}{|c|}{$* * *$} & \multicolumn{2}{|c|}{$* * *$} & \multicolumn{4}{|c|}{$* * *$} \\
\hline
\end{tabular}

a, b, c: Means with different superscript letters in the same column differ $(\mathrm{P}<0.05)$ for $0,4,8,12,16$, and $20 \mathrm{~h}$ at 6 wks of age, $\mathrm{X}^{2}$ : Chisquare value, $* * *: \mathrm{P}<0.001$.

\section{Discussion and Conclusion}

In line with a previous study $(7,15,20,26)$, cooled perch has statistically significant effect on BW on d 28, 35, 42 and FCR at 0-35 d. It is said that perch cooling helped birds in coping with heat stimulation. It is determined that BW at 35 and $42 \mathrm{~d}$, and FCR at $0-42 \mathrm{~d}$ was higher for the broilers reared at $1 \mathrm{~cm}$ litter thickness than those reared at 7 and $14 \mathrm{~cm}$ litter, although the difference between litter groups in terms of FCR at 0-42 d was not statistically significant. The results were found similar to those reported by Demirulus (4) $(5,8$, and $11 \mathrm{~cm}$ litter thickness), and Shao et al. (23) (4, 8, 12, and $16 \mathrm{~cm}$ litter thickness). In line with previous a study (6), $1 \mathrm{~cm}$ litter thickness was favourable for broiler performance, especially during the summer. In terms of BW of broilers, it can be said that thinner litter would increase foot pad and hock lesions (As in our unpublished data), and that decreased activity, physical exercise and perching frequency would improve BW gain. Results showed that carcass and carcass part weights were not significantly affected by cooled perch. Similarly, Feddes et al. (9), Quentin et al. (20) and Zhao et al. (26) reported that it is more difficult to modify carcass characteristics than to change growth performance. However, carcass and total breast weights were found higher in cooled perch group than non-cooled and no perch groups. As parallel to this finding, significant results of increased BW stimulated by cooled perch reflected some beneficial effects in carcass and breast weights were consistent with other studies (7, 25). It is thought that perch cooling did promote bird activity and bird mobility, leading to lower total legs and thigh meat weights. On the other hand, cooled perch would be very helpful for decreasing the body temperature by means of both perching and contact with wings or body, resulting in greater breast meat yield. It was determined that the highest carcass, total breast, and drums weights were $(2006.71,623.71$, and $282.19 \mathrm{~g}$, respectively) in the $1 \mathrm{~cm}$ litter thickness group. These findings were in consistent with other a study reporting that increases in carcass weights because of the thinnest litter group $(5 \mathrm{~cm})(4)$. Hence, decreasing litter thickness likely offered a more suitable litter for birds and increased some processing weights. It was determined that cooled perch has statistically significant effect on $\mathrm{pH}_{15}$ and $\mathrm{L}^{*}$ values from meat quality traits in thigh meat. The effect of cooled perch on meat quality characteristics is little studied in broilers. Zhao et al. (26) was reported that the lowest CL value $(27.1 \%)$ was determined in the cooled perch group, the highest CL value (31.8\%) was found in no perch group $(\mathrm{P}<0.05)$. Feng et al. $(10)$ reported that revealing that cyclic high temperatures induced lower $\mathrm{pH}$ and increased $\mathrm{L}^{*}$ value. Bao et al. (2), and Sandercock et al. (22) indicated a significant decrease in initial $\mathrm{pH}$ of breast muscle from 1 to 5 hours of heat exposure, compared with the control group. The $\mathrm{pH}$ value is a direct response of muscle acid content, resulting effects WHC and color of meat (5). Our findings suggest thigh meats of the birds in cooled perch had significantly higher lightness (56.96), compared with those in no perch group (62.54) $(\mathrm{P}<0.05)$. It was reported that higher $\mathrm{L}^{*}$ value for the broilers reared at $34^{\circ} \mathrm{C}$ than those reared at $21^{\circ} \mathrm{C}(14)$. It has been said that the increased $\mathrm{pH}_{15}$ and decreased $\mathrm{L}^{*}$ value found here with cooled perch indicates their utility effect on meat quality and results from improved 
thermolysis rather than perching over cooled perch and contact with cooled perch. In the present study, the $\mathrm{pHu}$ values of breast meat were lower than the $\mathrm{pHu}$ values of thigh meat. These differences can be explained by inactive breast muscles that had a more accelerated rate $\mathrm{pH}$ decline than active thigh muscles during the development of postmortem rigor mortis.

It was determined that the highest perching incidence in non-cooled and cooled perch treatments were 9.3 and $5.3 \%$, respectively, and the highest perching frequency in 1, 7, and $14 \mathrm{~cm}$ litter groups were 10.3, 6.9 and 3.3\%, respectively. Similarly, Hughes and Elson (12) reported that the frequency of perching was found $<27 \%$ of 8 wks broilers perching. Parallel to the current study, Zhao et al. (27) also indicated that perching frequency in broilers was found ranging from 7.47 to $12.8 \%$. Besides, in some studies $(7,13,19,24)$ was found the perching frequency in birds much lower (1.0-2.6\%) than our findings. These beneficial effects in perching frequency can be attributed to more activity by perch in the pen. And, the various perch types used in different studies likely explains these different findings. The perching frequency was found greater in $1 \mathrm{~cm}$ litter thickness group than $14 \mathrm{~cm}$ thickness group at 6 wks of age. If there was a prolonged contact with thinnest litters, feet health was adversely affected and growing sensitivity at feet could probably lead to FPD and HB (As in our unpublished data). Thus, the more frequent perching means the more reduced contact of the feet with the floor. Perch-contacting behaviour frequency was the highest $(41.7 \%)$ at 00:00 and 16:00 $\mathrm{h}$ in the $14 \mathrm{~cm}$ litter thickness group, whereas the highest proportion (25.1\%) of chickens in contact with perch were detected in broilers kept in $1 \mathrm{~cm}$ litter group for 00:00 h at 6 wks of age. The results indicated that perch-contact frequency was greater in the cooled perch group than non-cooled perch. These findings were in consistent with another study reporting that if given a choice, birds look for the cooling perch because it favoured heat transfer from the body of broilers to the cooled perch by conduction (7). The higher perchcontacting incidence was generally obtained in $14 \mathrm{~cm}$ litter thickness group, compared with $1 \mathrm{~cm}$ litter group. This result may be explained by the fact that it is not ideal to increase the thickness of litter, presumably due to depressed thermolysis from the significantly less heat elimination from the body by conduction, because of the reduction in contact with the feet on the ground.

Results indicated that broilers have higher tendency for using non-cooled perches than cooled perches at high ambient temperatures. Also, it is revealed that they preferred cooled perches than non-cooled ones for direct contact. It is also understood that cooled perches were very helpful for performance parameters, carcass characteristics, some meat quality traits, and perching. The results showed that $1 \mathrm{~cm}$ litter thickness has a favorable effect on growth performance, carcass weight, and total breast and drums weights of broilers reared at high temperatures. Potential favorable effects on bird welfare and litter quality of perch cooling in broilers raised in thicker litter need to be further investigated.

\section{Financial Support}

This study was supported by the Aydin Adnan Menderes University Research Foundation (Project number: VTF 17-053).

\section{Ethical Statement}

This study was approved by the Aydin Adnan Menderes University Animal Experimentation Local Ethics Committee (13.06.2017-64583101/049).

\section{Conflict of Interest}

The authors declared that there is no conflict of interest.

\section{References}

1. AOAC (1990): Official Methods of Analysis. Association of Official Analytical Chemists, Arlington, VA, USA.

2. Bao ED, Gong YY, Hartung J, et al (2004): Relation between pathologic damages and HSP70 changes in acute heat stressed broilers. Sci Agric Sinica, 37, 301-305.

3. Barton-Gade PA, Demeyer D, Honikel KO, et al (1993): Reference methods for water holding capacity in meat and meat products: Procedures recommended by an OECD working group. In: Proceedings of the $39^{\text {th }}$ International Congress of Meat Science and Technology, Calgary, Canada.

4. Demirulus H (2006): The effect of litter type and litter thickness on broiler carcass traits. Int J Poult Sci, 5, 670672.

5. El-Moniary MMA, Hemid AA, El-Wardany I, et al (2010): The effect of early age heat conditioning and some feeding programs for heat-stressed broiler chicks on: 1Productive performance. World J Agric Sci, 6, 689-695.

6. Erensayın C (1991): A Research on Technical Structure and Application of Commercial Poultry Farms in Turkey. Cumhuriyet University, Tokat Agriculture Faculty (Impress), Tokat, Turkey.

7. Estevez I, Tablante N, Pettit-Riley RL, et al (2002): Use of cool perches by broiler chickens. Poult Sci, 81, 62-69.

8. EU Commission Regulation (2007): No 2007/43/EC of 28 June 2007 on laying down minimum rules for the protection of chickens kept for meat production. Available at https://eur-lex.europa.eu/legal-content/EN/TXT/?uri= CELEX\%3A32007L0043. (Accessed November 2, 2019).

9. Feddes JJR, Emmanuel EJ, Zuidhoft MJ (2002): Broiler performance, body weight variance, feed and water intake, and carcass quality at different stocking densities. Poult Sci, 81, 774-779.

10. Feng JH, Zhang MH, Zheng SS, et al (2006): The effect of cyclic high temperature on mitochondrial ROS production, Ca2+-ATPase activity and breast meat quality of broilers. Chinese J Anim Vet Sci, 37, 1304-1311. 
11. Honikel KO (1998): Reference methods for the assessment of physical characteristics of meat. Meat Sci, 49, 447-457.

12. Hughes BO, Elson HA (1977): The use of perches by broilers in floor pens. Br Poult Sci, 18, 715-722.

13. LeVan NF, Estevez I, Stricklin WR (2000): Use of horizontal and angled perches by broiler chickens. Appl Anim Behav Sci, 65, 349-365.

14. Lu Q, Wen J, Zhang $H$ (2007): Effect of chronic heat exposure on fat deposition and meat quality in two genetic types of chicken. Poult Sci, 86, 1059-1064.

15. Meluzzi A, Fabbri C, Folegatti E, et al (2008): Effect of less intensive rearing conditions on litter characteristics, growth performance, carcass injuries and meat quality of broilers. Br Poult Sci, 49, 509-515.

16. Muiruri HK, Harrison PC (1991): Effect of roost temperature on performance of chickens in hot ambient environments. Poult Sci, 70, 2253-2258.

17. Nicol CJ (2015): The Behavioural Biology of Chickens. CABI, Oxfordshire.

18. Olsson IAS, Keeling LJ (2002): The push-door for measuring motivation in hens: laying hens are motivated to perch at night. Anim Welfare, 11, 11-19.

19. Pettit-Riley R, Estevez I (2001): Effects of density on perching behavior of broiler chickens. Appl Anim Behav Sci, 71, 127-140.

20. Quentin M, Bouvarel I, Berri C, et al (2003): Growth, carcass composition and meat quality response to dietary concentrations in fast-, medium and slow-growing commercial broilers. Anim Res, 52, 65-77.
21. Reilly WM, Koelkebeck KW, Harrison PC (1991): Performance evaluation of heat-stressed commercial broilers provided water-cooled floor perches. Poult Sci, 70, 1699-1703.

22. Sandercock DA, Hunter RR, Nute GR, et al (2001): Acute heat stress-induced alterations in blood acid-base status and skeletal muscle membrane integrity in broiler chickens at two ages: Implications for meat quality. Poult Sci, 80, 418-425.

23. Shao D, He J, Lu J, et al (2015): Effects of sawdust thickness on the growth performance, environmental condition, and welfare quality of yellow broilers. Poult Sci, 94, 1-6.

24. Su G, Sørensen P, Kestin SC (2000): A note on the effects of perches and litter substrate on leg weakness in broiler chickens. Poult Sci, 79, 1259-1263.

25. Zhao JP, Chen JL, Zhao GP, et al (2009): Live performance, carcass composition, and blood metabolite responses to dietary nutrient density in two distinct broiler breeds of male chickens. Poult Sci, 88, 2575-2584.

26. Zhao JP, Jiao HC, Jiang YB, et al (2012): Cool perch availability improves the performance and welfare status of broiler chickens in hot weather. Poult Sci, 91, 1775-1784.

27. Zhao JP, Jiao HC, Jiang YB, et al (2013): Cool perches improve the growth performance and welfare status of broiler chickens reared at different stocking densities and high temperatures. Poult Sci, 92, 1962-1971. 